\title{
Designing ad hoc temperature fixed point cells
}

\author{
Orest Kochan ${ }^{1, *}$, Jun $\mathrm{Su}^{2}$, and Roman Kochan ${ }^{1,3}$ \\ ${ }^{1}$ Lviv Polytechnic National University, Department of measuring information technologies, 79013 Karpinskoho Str. 1, Lviv, \\ Ukraine \\ ${ }^{2}$ School of Computer Science, Hubei University of Technology, Wuhan, China \\ ${ }^{3}$ University of Bielsko-Biala, Bielsko-Biala, Poland
}

\begin{abstract}
The design of the ad hoc (made of hand-on materials) temperature fixed-point cell (TFPC) is considered in this paper. There was developed a TFPC filled with lead and an apparatus for its investigation. The feature of the considered TFPC is minimization of the stray thermal fluxes distorting the temperature plateau during a phase change. Conclusions about the influence of the design of a TFPC on the temperature plateau of phase change are described.
\end{abstract}

\section{Introduction}

According to [1], the share of temperature measurements among all measurements in industry is $40 \%$, but in energetics this percentage is about $70 \%$. The accuracy of temperature sensors in most cases is decisive for highquality production [2-4]. Also, a proper temperature mode is crucial for durable operation and safety of equipment [5-8]. Thus, the accuracy of measurements has a considerable effect on the world economy [8]. The estimation of the market of metrological services is approximately $4 \%$ of GDP in developed countries [9]. The progress in metrology means accuracy increase and development of techniques that increase metrological reliability of measurements as well as implementation into praxis new metrological procedures and instruments that improve metrological service [6,7]. The progress in temperature measurements is important too [10]. Its accomplishments can give a considerable economic gain. For instance, the increase of temperature measurement accuracy in power plants will gain $\$ 400$ million worldwide [8] with no additional expenses of fuel. The analysis of literature $[1,11,12]$ shows that accuracy of measuring instruments is constantly growing up $[13,14]$, however, the error of temperature measurements has decreased very little because the error of sensors dominates in temperature measuring chains $[1,11,12]$. Despite all efforts in recent decades, the error of sensors has decreased very little.

Thermocouples (TCs) are the most widely used sensors for temperature measurements above $650^{\circ} \mathrm{C}$, despite the fact that their error is much bigger than that of the rest of the measuring chain $[1,11,12]$.

At first glance, it is reasonable to make the operation time as long as possible, because it saves the sensor itself and cuts down the expenses connected with a sensor replacement. However, this is connected with losses due to big measurement error. On the other hand, it is not always possible to ensure metrological service in-situ to decrease the error of a sensor.

During long-term operation of a TC, its legs become inhomogeneous [15-17] under the influence of time and operation temperature. The rate of inhomogeneity strongly depends on operating conditions, thermal shocks, vibration, contaminations, etc. There is no direct method for determining the degree of TC inhomogeneity. This inhomogeneity is the main source of error when measuring a temperature by thermocouples $[15,16]$. It changes the Seebeck coefficient of a TC sections. Thus, the Seebeck emf of the TC will drift with time in accordance with the change of inhomogeneity in the TC legs, therefore the error appears due to the deviation of the actual conversion characteristic of a TC from the nominal one.

The traditional method for determining the error of TCs is a periodic calibration in a calibration laboratory [17]. However, under laboratory conditions the error of TCs is very likely to be different than in-situ $[17,18]$. In addition, this procedure usually requires the complex multistep procedure: stop of operation, removal of measuring equipment from the operating place, transfer it to the calibration laboratory, calibration, return the measuring equipment back to the operating place, installation of equipment again and launch the operation again. The methods for calibration of TC in situ seem to be the best ones. Therefore, recently a lot of attention has been paid to developing the temperature fixed-point cells (TFPCs) $[10,19]$ for in situ calibration.

The TFPC is a capsule containing a reference material [20] and is put on the measuring junction of the TC to determine the thermocouple error during melting or freezing. Reference materials are pure metals or eutectic alloys whose temperatures of phase change are accurately known and remain constant during the phase change [21]. This constant temperature of the phase change is called "a temperature plateau" (Fig. 1): a- the

\footnotetext{
Corresponding author: orestvk@gmail.com
} 
plateau while melting a reference material; b - the plateau while freezing; c - the plateau while freezing with supercooling. The temperature of a plateau is known with high accuracy and can be converted into emf [1]. Comparison of this emf with the one measured by a TC allows determining the TC error. It is reasonable to carry out this procedure in situ. TFPCs are not yet widely used in industry because of the technological problems.

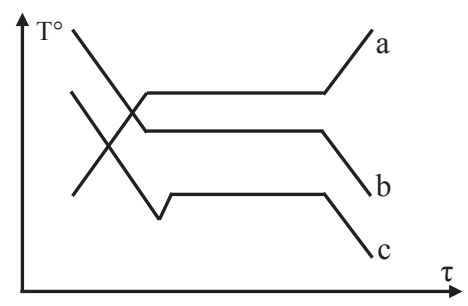

Fig. 1. Different types of a temperature plateaus.

They are usually used in laboratories. However, in the last decade there appeared many publications in leading journals and reports on conferences in this area. Such interest of researchers to TFPCs gave experts the reason to predict a significant increase of TFPCs usage in the range of medium and high temperatures $[10,19]$.

\section{State of the art}

The typical implementation of a TFPC is described in [20]. Fig. 2 shows the change of Seebeck emf in time in modes of sustainable heating and cooling as given in [20]. Fig. 2 demonstrates that the temperature plateau at the phase change is difficult to identify. Furthermore, it is clear that the temperature of melting is not equal to that of freezing. It should be noted that this type of a temperature plateau (Fig. 2) directly contradicts physics [21], indicating that the temperatures of melting and freezing are, stable and equal. The graphs [20] can be explained by the presence of a significant stray thermal flux (Fig. 3) in the studied TFPC, which heats the measuring junction of the TC while melting (when the environment temperature is above than that of the phase change) and cools while freezing (when the temperature is below than that of the phase change).

The stray thermal flux distorting the temperature plateau is shown in Fig. 3 with dashed lines. It does not come across the phase change boundary of the reference material [20]. Therefore, the temperature of the measuring junction of the TC does not equal to that of the phase change. Thus, the increase in the accuracy of the temperature measurements using the TFPC described in [20] is doubtful. Thus, as seen from the analysis, the main cause of the poor temperature plateau in a TFPC may be its ineffective design. Designs of the known TFPCs are different [22-24], but many of them have the similar drawbacks.

The theory of designing of TFPCs, including calculation of thermal processes in a TFPC, is proposed in [24], where the basic features of their designs are explored. However, as seen in [20,22,23], structural factors are often not taken into account when designing a TFPC.

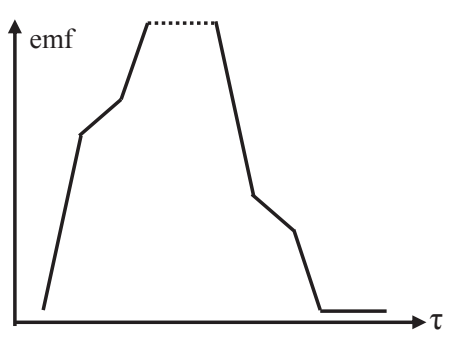

Fig. 2. The actual temperature plateau, [26]

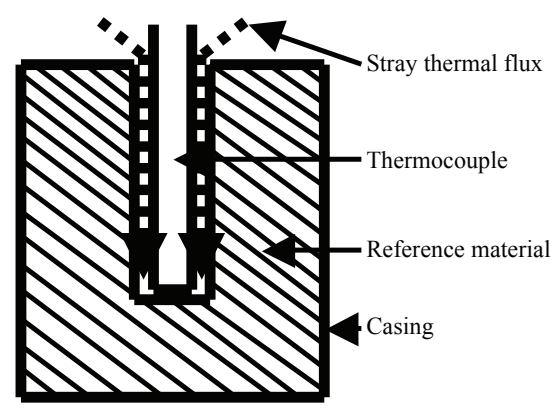

Fig. 3. The cross section design of the TFPC [25].

The aim of this paper is to design an ad hoc (made of improvised materials) TFPC to demonstrate that reduction of the stray temperature flux allows obtaining high quality temperature plateaus.

\section{Designing the TFPC}

In order to make the temperature plateau, that forms the Seebeck emf of the TC, accurately consistent with the phase change temperature of the TFPC, there should be reduced (preferably completely eliminated) the stray thermal flux that flows toward/outward the measuring junction of a TC from the outer environment (see. Fig. 3 ). The TFPC should ensure the adiabatic conditions for the measuring junction of a TC; heat exchange must take place between the TC measuring junction and a reference material, and between the reference material and the outer environment. Heat transfer between the TC measuring junction and the outer environment has to approach zero.

This can be achieved, by maximizing the coverage area of the TC measuring junction with the liquid reference material (at the temperature of phase change), and by maximizing the distance for the stray heat flux to flow. How to ensure these conditions is shown in Fig. 4. The TC measuring junction is deeply immersed into the reference material that covers it from all sides. When heating is sustainable, the medium (the furnace) temperature increases as the arrows show in Fig. 4. The TC with the TFPC have much higher heat capacity than the environment, thus the temperature of the TFPC is lower and the stray thermal flux flows in the way to heat the TFPC and the TC measuring junction. When the reference material begins to melt, the temperature of 
phase change remains constant. Then the thermal flux heating the TC is split into two ones. The first flux comes from the furnace and ensures the continuation of melting. The second flux goes from the surface of the phase transition to the TFPC center and the TC measuring junction. The latter thermal flux provides equalization of the TC temperature (see Fig. 4, the temperature change direction is indicated by the arrows within the surface of the phase change). The direct thermal flux from the furnace toward the TC measuring junction is only possible through the TC legs, but they should be long enough and heat exchange between them and the reference material should be good. Therefore, the stray thermal flux, which distorts the temperature plateau (Fig. 2 and 3), has a minimum value.

\section{The design of the proposed ad hoc TFPC}

The simple design and technique of designing the TFPC are offered. There were taken precautions to minimize the effect of the stray thermal flux on temperature plateau toward or outward the reference material.

Firstly, as it is shown in Fig. 5 there was prepared a TC. There was used type K TC made of chromel-alumel with $1.2 \mathrm{~mm}$ diameter of legs. It was covered with a long $(20 \mathrm{~cm})$ ceramic insulating bead with the outer diameter of $8 \mathrm{~mm}$. The legs between the bead and the measuring junction of the TC were bare for approximately $35 \mathrm{~mm}$. This area of the TC was covered with two thin layers of a mixture of sodium silicate (liquid glass) with gypsum. The total coating thickness was approximately $0.5 \mathrm{~mm}$.

After this, the TFPC was prepared. Its body was made of the copper tube with the inner diameter of $8 \mathrm{~mm}$ and its length was $100 \mathrm{~mm}$; it was folded from one side. A $35 \mathrm{~mm}$-long screw of $4 \mathrm{~mm}$ in diameter was twisted in with washers and nuts to cork the folded end. The tube was filled with finely chopped lead, which was then melted on a gas burner. The amount of lead was chosen in the way to fill the tube on $75 \ldots 80 \%$. Then the lead in the case was melted again and the TC was immersed into it with tension at the maximum possible depth (at least $20 \mathrm{~mm}$ ) (see. Fig. 5). The prepared TFPC with the immersed TC is shown in Fig. 6. The proposed design of the TC with TFPC for study corresponds to Fig. 4. The TC measuring junction is almost completely covered with the reference material. The TC legs are immersed into the reference material in about 20 their diameters and coated with a thin layer of insulation, i.e. they have good thermal contact with the reference material. The ratio of the case length of the TFPC to its diameter is about 10, so according to [26], the case can be considered infinitely long. The stray thermal flux must have a minimum effect on the temperature plateau. The TC measuring junction is like in a "thermos" that stores inside the TFPC the phase change temperature regardless of changes in temperature in the furnace [25]. It should be noted that the above considerations about the form of the temperature plateau in the proposed design of the TFPC, are mainly qualitative, therefore, they require experimental confirmation.

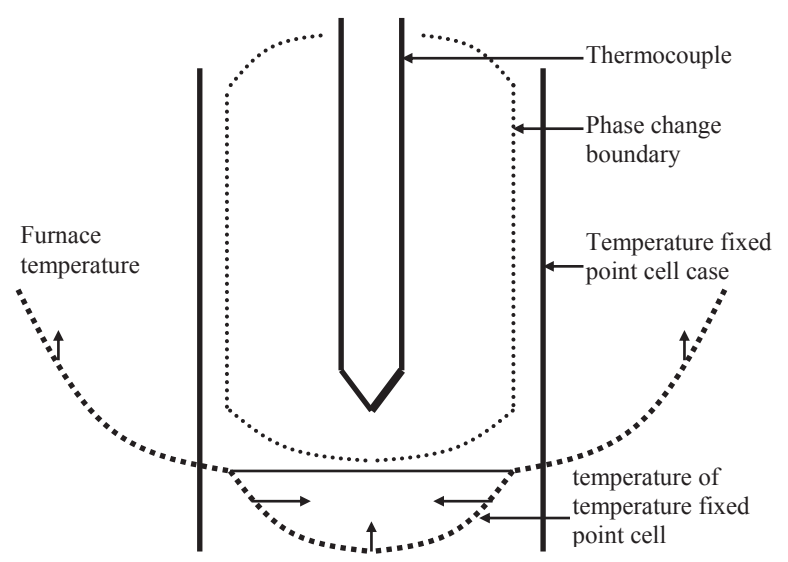

Fig. 4. The proposed TFPC and its thermal fluxes.

\section{The apparatus to study of the proposed TFPC}

There was constructed an apparatus for experimental studies of the proposed design of the TFPC. The main element of the apparatus is a shaft furnace, made of a tubular wire resistor of type PEV with the nominal power rating of 50 watts. It is located in the center of a glass jar which then is filled with gypsum. Power circuit consists of an autotransformer of type LATR-1, the output voltage is measured with the analog multimeter of type C20. The temperature of the furnace was measured with the additional type K TC. The temperature measurement of the reference junctions of both TCs was carried out using a thermistor of type KMT4 with the nominal resistance of $30 \mathrm{k} \Omega$. It was previously individually calibrated using the mercury thermometer of type TL18 (its error does not exceed $0.1^{\circ} \mathrm{C}$ ). The tested TFPC with the TC was placed in the center of the furnace. The distance to the bottom of the furnace was set using the $35 \mathrm{~mm}$-long screw of $4 \mathrm{~mm}$ in diameter and a centering plate was fixed at its end. To reduce the temperature gradient in the furnace and temperature changes of the reference junctions the following steps were taken: 1) the furnace is closed with a plaster lid with holes for both TCs; 2) the lid is sealed additionally with plaster solution and covered with cotton wool; 3) the reference junctions of both TCs and the thermistor are placed in a copper box; 4) the copper box is covered with a layer of cotton wool. The measuring system includes a TC equipped with the TFPC, a TC measures the temperature in the furnace, the thermistor Rt to measure the temperature of the two reference junctions of the TCs, the switch S1 for the TCs, the switch S2 between the TCs and the thermistor as well as the precision digital multimeter (DMM) of type UT70D. When measuring the Seebeck emf, the UT70D operates in the mode of voltage measurement under improved resolution (the resolution of $1 \mu \mathrm{V}$ corresponding to approximately $0.025^{\circ} \mathrm{C}$ for a type $\mathrm{K} \mathrm{TC}$ ). When measuring the resistance of the thermistor with the nominal resistance of $30 \mathrm{k} \Omega$, the UT70D operates in the resistance measurement mode with the resolution of $1 \Omega$, which corresponds to approximately $0.0012^{\circ} \mathrm{C}$. It should be noted that the instability of the temperature during the temperature plateau while melting or freezing of the reference material is calculated as the difference between the current temperature and the initial 
temperature (the temperature at the point, which is assigned to be the beginning of the phase change).

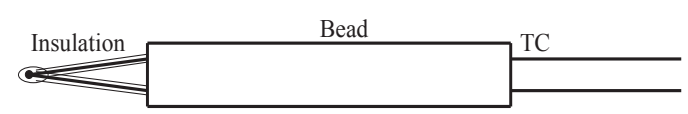

Fig. 5. The TCprepared for immersion into a TFPC.

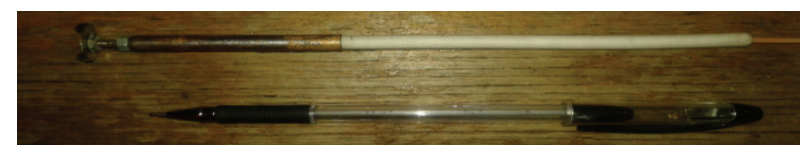

Fig. 6. TC with a TFPC.

In this case, the systematic errors of the measuring system, including the TCs and the DMM will not distort temperature plateau. Thus, only the random errors of the measurement circuit affect the measurement error of the Seebeck emf instability of the temperature plateau. The study before the experiments showed that this error does not exceed $\pm 1 \mu \mathrm{V}$. The systematic measurement error of the reference junction temperature is also compensated by the fact that the purpose of the experiment is to measure the temperature change during the phase change of the reference material. The random error of the correction of the reference junction temperatures, when the resolution of the thermistor resistance measurement is of $0.0012^{\circ} \mathrm{C}$, can be neglected.

\section{Conclusions}

When designing a TFPC stray thermal flows have to be taken into account to keep the slope of a temperature plateau of the order of a few tenth of a Celsius degree to fulfil the European roadmap for thermometry [10]. As has been shown in this paper, the proper design of the TFPC, which prevents the heat exchange between the reference material and environment, makes it possible to achieve high quality temperature plateau even for a relatively simple ad hoc TFPC made of on hand materials. Such ad hoc TFPCs can be used for calibration of TCs in situ.

The study of metrological parameters of a TFPC (in particular, the stability of the phase change temperature) makes sense only when a TFPC ensures a high quality temperature plateau.

This work was supported by Foundation of Wuhan Science and technology Bureau (2015030809020370), Doctoral Scientific Research Fund from Hubei University of Technology (No. BSQD14037), National Natural Science Foundation of China (Nos. 61602162, 61502155), Green Industry Technology Leading Project of Hubei University of Technology (No. ZZTS2016004).

\section{References}

1. Y. Lutsyk, O. Hook, O. Lakh, B. Stadnyk, Temperature Measurements: Theory and Praxis (Lviv, 2006) (In Ukrainian)

2. A. Glowacz, Z. Glowacz, Appl. Acoust. 117 (Part A), 20-27 (2017)

3. P. Hreha, A. Radvanska, L. Knapcikova, G.M. Krolczyk, S. Legutko, J.B. Krolczyk, Hloch S.,
Monka P., Metrol. Meas. Syst. 22, 2, 315-326 (2015)

4. V. Perzel, M. Flimel, J. Krolczyk, A. Sedmak, A. Ruggiero, D. Kozak, A. Stoic, G. Krolczyk, S. Hloch, Thermal Sci. 00, 46 (2016) DOI: 10.2298/TSCI150212046P

5. H. R. Ashrafi, K. Shahbazian, S. Bidmeshki, S. Yaghooti, P. Beiranvand. Adv. Sci. Technol. Res. J. 10, 29, 1-12 (2016)

6. A. Glowacz, Z. Glowacz, Measurement, 93, 86-93 (2016)

7. J. Su, O. Kochan, R. Kochan, Int. J. Thermop. 37, 4, 1 (2016)

8. T. Wang et all, Stabilized metal sheathed type K and E thermocouples improve turbine efficiency, ISA tech/expo technology update, 1, 5, 439-448 (1997)

9. J. Birch, Benefit of legal metrology for the economy and society, (A study for the International Committee of Legal Metrology, 2003)

10. G. Machin, et all. Int. J. Thermop. 35, 3-4, 21-30 (2014)

11. J. Webster, Measurement, Instrumentation, and Sensors Handbook (CRCnetBase, 1999)

12. P.R.N. Childs, Practical Temperature Measurement (Elsevier, 2001)

13. A. Aly, A. El-Lail, I.J. Inf. Technol. and Comput. Sci. 03, 56-60 (2013)

14. http://www.thermoelectra.com/en/Products/Instruments

15. D.J. Southworth, Temperature Calibration with Isotech Block Baths (Southport, Isotermal technology ltd, 1999)

16. K. C. Sloneker, Ceram. Ind. 159, 4, 13-18 (2009).

17. J. Su, O. Kochan, Meas. Tech+ 57, 10, 1160-1166 (2015)

18. S. Chang, O. Kochan, Sensors \& Transducers 160, 12, 55-61, (2013)

19. G. Machin, Twelve years of high temperature fixed point research: a review, Temperature: Its Measurement and Control in Science and Industry, 8(1), 305-316 (2013)

20. O. Ongrai, J.V. Pearce, G. Machin, S. Sweeney, Meas. Sci. Technol. 22, 015104 (2011)

21. D. Halliday, Fundamentals of Physics (Wiley, 2011)

22. S. Rudtsch, A. Aulich, C. Monte, Stray thermal influences in zinc fixed-point cells. Temperature: Its Measurement and Control in Science and Industry, 8, 1, 265-270 (2013)

23. M. Kalemci, et al., Int. J Thermophys. 36, 19681979 (2015)

24. A. Sachenko, V. Milchenko, V. Kochan, Measuring Temperature Using Sensors with Built-In Calibrators (Moscow, 1986)

25. S. Chang, O. Kochan, et all, Demands to the design of temperature fixed point cells, Proc. of the workshop TEMPMEKO, 244 (2016)

26. J.IV Lienhard, and J.V. Lienhard, A Heat Transfer Textbook (Cambridge: Phlogiston Press, 2008) 